\title{
Hydrocalyx: Uncommon Complication of Percutaneous Nephrolithotomy
}

\author{
Mustafa Sungur1, Selahattin Caliskan² and Utku Lokman³
}

\begin{abstract}
The success rate of percutaneous nephrolithotomy (PNL) is about $90 \%$, but overall complications of this surgery have been reported in up to $83 \%$ cases. The complications are graded using Clavien classification system from grade 1 to 5 . Grade 1 needs simple treatments without surgical, endoscopic, and radiologic interventions like antiemetics, antipyretics, and analgesics. Grade 5 denotes death of the patient. Calyx neck stricture is grade $3 b$ complication with an incidence of $0.1 \%$. In this study, a case of hydrocalyx, which is very rare complication after PNL, is presented. To the best of the authors' knowledge, there is no hydrocalyx case reported after PNL in the literature.
\end{abstract}

Key Words: Hydrocalyx, Percutaneous nephrolithotomy, Clavien classification system.

\section{INTRODUCTION}

Percutaneous nephrolithotomy (PNL) is defined as a minimally invasive method for renal stones and has replaced open stone surgery in most of the patients, since 1970's. PNL is the first treatment option for renal calculi larger than $20 \mathrm{~mm}$. This surgical procedure has some advantages such as high success rate, short healing period, a small incision and absence of the scar. Like every surgical procedure, PNL has also specific complications. Bleeding necessitating intervention, injuries to the adjacent organs, urinary tract injury, and death are the major complications. Minor complications, such as postoperative fever, bleeding necessitating transfusion, extravasation, tube dislodgement, pneumonia, and prolonged urine drainage may be seen in more than $50 \%$ of the patients. ${ }^{1}$ Hydrocalyx is an uncommon complication of PNL.

Here, we present a case of a man who was treated with PNL 10 years ago and now presented with giant renal cyst formation with residual stones.

\section{CASE REPORT}

A 52-year man presented to the institute clinic with right dull flank pain for 2 years. In his part history, he had open surgery for left renal stone 15 years ago and PNL for right renal stone 10 years ago. He had a computed

1 Department of Urology, Hitit University, Erol Olcok Training and Research Hospital, Corum, Turkey

2 Department of Urology, Kanuni Sultan Suleyman Training and Research Hospital, Corum, Turkey

3 Department of Urology, Faculty of Medicine, TOBB Economy and Technology University, Corum, Turkey

Correspondence: Dr. Mustafa Sungur, Department of Urology, Hitit University, Erol Olcok Training and Research Hospital, Corum, Turkey

E-mail:sevmus2005@gmail.com

Received: January 29, 2018; Accepted: July 30, 2018 tomography $(\mathrm{CT})$ before $\mathrm{PNL}$, showing a $2 \mathrm{~cm}$ renal pelvic stone and multiple lower calyx stones, the largest one being $1 \mathrm{~cm}$. There was no abnormality in the pelvicalyceal anatomy and no cystic diseases before PNL.

Urine microscopy was normal and the culture was sterile. Laboratory results were within normal limits; creatinine and blood urea nitrogen were 1.1 and 25 $\mathrm{mg} / \mathrm{dl}$, respectively.

Ultrasonography showed an $84 \times 95 \mathrm{~mm}$ renal cystic lesion, in the lower pole of the right kidney. CT had also revealed the $10 \times 9 \mathrm{~cm}$ sized right renal cyst and multiple stones, the largest one $8-9 \mathrm{~mm}$ in the cyst (Figure 1). Magnetic resonance imaging (MRI) demonstrated right lower pole cyst with a size of $9.5 \mathrm{~cm}$. Finally, right retrograde pyelography was performed and the images showed the transition of minimal contrast medium to the enlarged body, suggested as hydrocalyx. No functional renal parenchyma was present around the cyst.

The patient was treated with transperitoneal laparoscopic renal surgery. After entering the peritoneum, right kidney was dissected and lower pole identified. The giant hydrocalyx of the right kidney was aspirated and

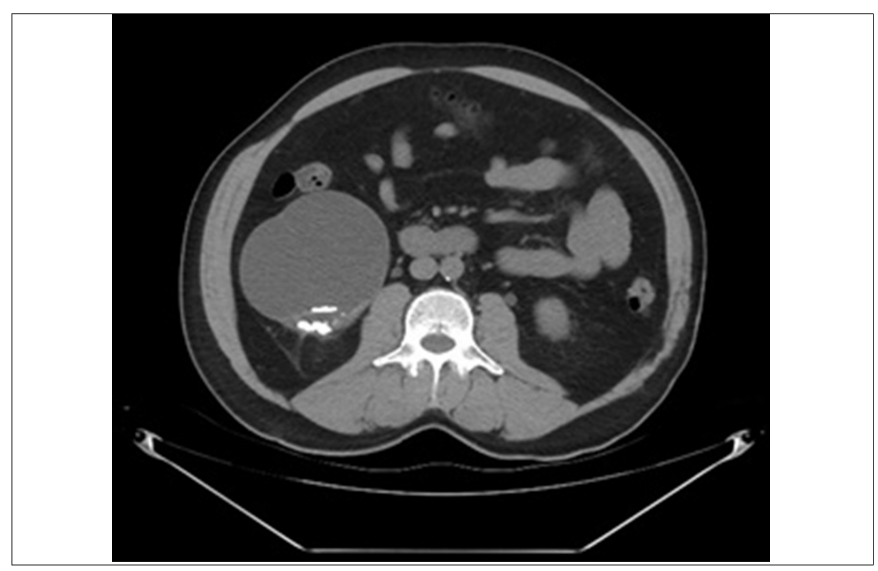

Figure 1: CT scan of the hydrocalyx. 


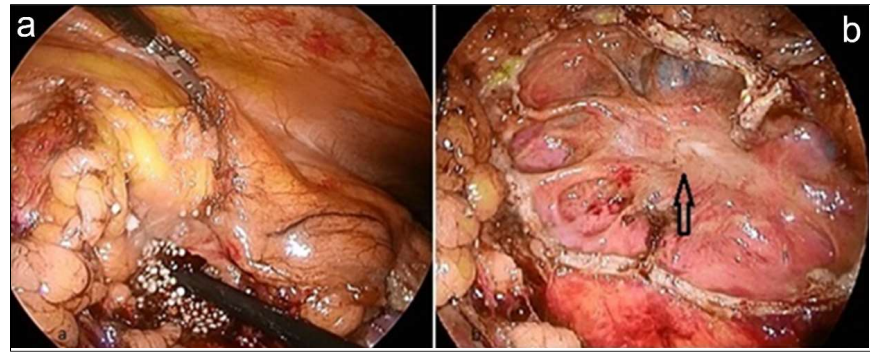

Figure 2: (a) Stone fragments are shown in the hydrocalyx; (b) after cleaning the stones from the hydrocalyx (arrow shows the calyx neck).

excised with a harmonic scalpel (Ethicon USA). Residual multiple stone fragments were cleaned and calyx neck was identified (Figures $2 a$ and $2 b$ ). After excision of the cyst wall, the calyx neck on the base of the cyst was clearly delineated and sutured with $4 / 0$ vicryl sutures. After that an absorbable hemostat material was placed on the calyx neck. The remaining Gerota fascia was also placed on this calyx neck, in order to prevent any leakage. The pathology of the specimen was chronic pyelonephritis with atrophic changes in the renal parenchyma. The patient had no complaints during the 18 months follow-up period.

\section{DISCUSSION}

Although PNL has a high success rate, overall complications during or after PNL may be seen in up to $83 \%$ of cases. ${ }^{2}$ The modified Clavien grading system is a valuable classification for perioperative and postoperative complications of PNL. There are five grades; from any deviation of normal postoperative course without the need for treatment to death.

However, the rates of the complications differ in the literature, most of them are clinically insignificant bleeding or fever and resolve without intervention. The frequency of major complications is low $0.9-4.7 \%$ for septicemia and $0.6-1.4 \%$ for renal hemorrhage requiring intervention. ${ }^{2}$

After PNL, renal collecting system obstruction is very rare but may result from ureteral avulsion or stricture, transient mucosal edema, blood clot, or infundibular stenosis.3,4 Blood clot or edema can cause transient renal collecting system obstruction and often resolve without intervention or any long-term sequelae.
We could not find any hydrocalyx case after PNL in the literature; but in their review, Parsons et al. reported five cases of infundibular stenosis in the series of $223(2.2 \%)$ patients after PNL procedures. ${ }^{4}$ These five patients underwent more than one percutaneous stone removal procedure. The authors reported the risk factors for infundibular stenosis as prolonged operative time, large stone, multiple removal procedures, and extended postoperative nephrostomy tube drainage. In this case, at first, the mechanism probably was an infundibular stenosis, and the progressive obstruction caused the giant hydrocalyx.

Renal collecting system obstruction associated with ureteral stricture or avulsion can lead to nephrocutaneous fistulae, hydronephrosis or hydrocalyx. Facilitating factors are previous ipsilateral open pyelolithotomy, diabetes and morbid obesity. ${ }^{4}$ Early diagnosis and treatment of renal collecting system obstruction, significantly decreases complication rate. Retroperitoneal urinoma was reported after PNL operation secondary to the residual stone migration to the upper ureter. 5

Renal collecting system obstruction limited to infundibular stenosis may be treated with observation and endoscopic dilation. For severe and prolonged cases that cause hydrocalyx, the only choice is excision of the dilated part or heminephrectomy. ${ }^{4}$

In conclusion, PNL is an effective treatment for renal stones but complications should also be kept in mind. Hydrocalyx is a very rare complication of PNL and clinicians should be aware that the patients may present with hydrocalyx several years after surgery.

\section{REFERENCES}

1. Lee WJ, Smith AD, Cubelli V. Complications of percutaneous nephrolithotomy. AJR Am J Roentgenol 1987; 148:177-80.

2. Michel MS, Trojan L, Rassweiler JJ. Complications in percutaneous nephrolithotomy. Eur Urol 2007; 51:899-906

3. Segura JW, Patterson DE, LeRoy AJ. Percutaneous removal of kidney stones: Review of 1,000 cases. J Urol 1985; 134:1077-81.

4. Parsons JK, Jarrett TW, Lancini V. Infundibular stenosis after percutaneous nephrolithotomy. J Urol 2002; 167:35-8.

5. Çaliskan S, Çevik R. Retroperitoneal urinoma after percutaneous nephrolithotomy. Med Sci 2016; 5:720-4. 